International Mathematical Forum, 2, 2007, no. 68, 3385 - 3393

\title{
Convergence of Multigrid Algorithms Incorporating an Artificial Damping
}

\author{
Ali Tavakoli \\ Department of Mathematics \\ Ferdowsi University of Mashhad, Iran \\ alta640@yahoo.com \\ Asghar Kerayechian \\ Department of Mathematics \\ Ferdowsi University of Mashhad, Iran
}

\begin{abstract}
When the solution and coefficients in a problem are highly oscillatory, there exists sometimes a glaring difference between solution and physical results. This occurrence can be arisen by the fact that the coarse grid problem is still huge and poorly-conditioned. In this case, one way for solving such problems is to apply an artificial damping in multigrid algorithm. In [9], a multigrid algorithm incorporating an artificial damping has been used for solving a model problem, but the convergence proof has not been presented. In this paper, a multigrid algorithm is presented and the convergence of the algorithm is proved for a general problem.
\end{abstract}

Mathematics Subject Classification: 35JXX, 58J90

Keywords: Multigrid algorithms, Artificial damping

\section{Introduction}

Multigrid algorithms have been used extensively as tools for obtaining approximations to the solutions of partial differential equations. In conjunction, there has been intensive research into the theoretical understanding of these methods ([1]). When we solve a problem by multigrid method and the solution is oscillatory, then a coarse grid interpolation of space may not work well. In other words, the coarse grid problem may be still huge and poorly conditioned 
([6]). Using an artificial damping is a suggestion for solving such problems. In [9], it has been shown that a slight amount of artificial damping makes the coarse grid solver converge faster, without deteriorating the convergence rate of the original multigrid method. For instance, we can consider the following problem for a fine grid.

$$
\begin{aligned}
-\nabla \cdot(a \nabla u) & =f, \quad \text { in } \Omega \\
a \frac{\partial u}{\partial \nu}+\eta u & =0, \quad \text { on } \partial \Omega
\end{aligned}
$$

where $\Omega$ is a rectangular domain in two dimensions, $\partial \Omega$ is its boundary, $\nu$ is the unit outward normal on $\partial \Omega, 0<\eta_{1} \leq \eta(x, y) \leq \eta_{2}$ and $a$ is the diffusion coefficient.

A coarse grid problem for (1) including an artificial damping has been given by the following form:

$$
\begin{aligned}
-\nabla \cdot\left(a \nabla u_{\sigma}\right)+\sigma u_{\sigma} & =f, \quad \text { in } \Omega, \\
a \frac{\partial u_{\sigma}}{\partial \nu}+\eta u_{\sigma} & =0, \quad \text { on } \partial \Omega,
\end{aligned}
$$

where $\sigma \geq 0$ is the artificial damping (compressibility) coefficient.

Various convergence proofs for multigrid algorithms has been widely presented so far $([2,5])$. Attempts has been accomplished for improvement of convergence rates $([4,11])$. However, less work has been done on the convergence of multigrid algorithms including an artificial damping. In this paper, we present a multigrid algorithm incorporating an artificial damping and we shall prove its convergence. Of course, for proving we use the same assumptions that has been given in [2].

In Section 2, we use a two-grid algorithm including an artificial damping. This algorithm is a combination of symmetric multigrid algorithm in [2] and the two-grid algorithm incorporating an artificial damping in [9]. In this section also three conditions are stated. They are appropriate conditions for proving convergence of the multigrid algorithm. Conditions 1 and 2 are the same as those in [2]. In Section 3, we prove that for sufficiently small artificial damping, the two-grid algorithm given in Section 2 is convergent.

\section{Preliminary Notes}

We assume that $\mathcal{M}_{h}$ and $\mathcal{M}_{h_{c}}$ are nested finite-dimensional inner product spaces such that $\mathcal{M}_{h_{c}} \subset \mathcal{M}_{h}$.

In addition, let $(., .)_{h}$ and $(., .)_{h_{c}}$ be symmetric positive definite bilinear forms on $\mathcal{M}_{h}$ and $\mathcal{M}_{h_{c}}$ respectively. Let also $a(.,$.$) be symmetric positive$ definite bilinear form on $\mathcal{M}_{h}$ and $\mathcal{M}_{h_{c}}$. We shall develop two-grid algorithms for the solution of the following problem: Given $f \in \mathcal{M}_{h}$, find $u \in \mathcal{M}_{h}$ such that 


$$
a(u, v)=f(v) \quad \forall v \in \mathcal{M}_{h} .
$$

Let $A_{h}: \mathcal{M}_{h} \rightarrow \mathcal{M}_{h}$ be the discretization operator on level $h$ given by

$$
\left(A_{h} v, w\right)=a(v, w), \quad \forall w \in \mathcal{M}_{h}
$$

The operator $A_{h}$ is clearly symmetric in both the $a(.,$.$) and (., .)_{h}$ inner products and positive definite. Also, we define the discretization operators $A_{h_{c}}: \mathcal{M}_{h_{c}} \rightarrow \mathcal{M}_{h_{c}}$ and $H_{h_{c}}: \mathcal{M}_{h_{c}} \rightarrow \mathcal{M}_{h_{c}}$ by

$$
\left(A_{h_{c}} v, w\right)=a(v, w) \quad \forall w \in \mathcal{M}_{h_{c}},
$$

and

$$
\left(H_{h_{c}} v, w\right)=b(v, w) \quad \forall w \in \mathcal{M}_{h_{c}} .
$$

Now, we define the operator $\widetilde{A}_{h_{c}}: \mathcal{M}_{h_{c}} \rightarrow \mathcal{M}_{h_{c}}$ by $\widetilde{A}_{h_{c}}=A_{h_{c}}+\sigma H_{h_{c}}$ where $\sigma$ is a nonnegative real number.

The operators $A_{h_{c}}, H_{h_{c}}$ and $\widetilde{A}_{h_{c}}$ are symmetric in the $(., .)_{h_{c}}$ inner product and positive definite. Indeed, it is not hard to show that $A_{h_{c}}$ is symmetric in $a(.,$.$) inner product.$

We define the operators $P_{h_{c}}: \mathcal{M}_{h} \rightarrow \mathcal{M}_{h_{c}}$ and $P_{h_{c}}^{0}: \mathcal{M}_{h} \rightarrow \mathcal{M}_{h_{c}}$ by

$$
a\left(P_{h_{c}} v, w\right)=a(v, w), \quad \forall w \in \mathcal{M}_{h_{c}},
$$

and

$$
\left(P_{h_{c}}^{0} v, w\right)=(v, w), \quad \forall w \in \mathcal{M}_{h_{c}} .
$$

Note that $P_{h_{c}}$ is symmetric in the $a(.,$.$) inner product.$

Also, we require a linear smoothing operator $R_{h}: \mathcal{M}_{h} \rightarrow \mathcal{M}_{h}$. We assume that $R_{h}$ is symmetric in the $(., .)_{h}$-inner product and set $K_{h}=I-R_{h} A_{h}$. We further assume that $K_{h}$ is nonnegative in the sense that $a\left(K_{h} u, u\right) \geq 0$ for all $u \in \mathcal{M}_{h}$.

Now, we are ready to define the two-grid algorithm incorporating an artificial damping as follows.

\section{Two-Grid Algorithm}

Assume that $\sigma$ is a nonnegative artificial damping and $\widetilde{A}_{h_{c}}=A_{h_{c}}+\sigma H_{h_{c}}$. Set $B_{h_{c}}=\left(\widetilde{A}_{h_{c}}\right)^{-1}$. Define $B_{h} g$ for $g \in \mathcal{M}_{h}$ as follows:

(1) Set $x^{0}=0$.

(2) Define $x^{l}$ for $l=1, \cdots m$ by

$$
x^{l}=x^{l-1}+R_{h}\left(g-A_{h} x^{l-1}\right) .
$$


(3) Define

$$
x^{m+1}=x^{m}+B_{h_{c}} P_{h_{c}}^{0}\left(g-A_{h} x^{m}\right) .
$$

(4) Define $x^{l}$ for $l=m+2, \cdots, 2 m+1$ by

$$
x^{l}=x^{l-1}+R_{h}\left(g-A_{h} x^{l-1}\right) .
$$

(5) Set $B_{h} g=x^{2 m+1}$.

In this algorithm, $m$ is a positive integer which may vary from level to level and determines the number of pre and post smoothing iterations.

Now, we require appropriate conditions for the smoother, need to establish the stability property of the intergrid transfer operator and show the so-called " regularity and approximation " inequality. Below we use $\|u\|=(u, u)^{\frac{1}{2}}$ and $\|u\|_{a}=a(u, u)^{\frac{1}{2}}$.

The conditions which were often assumed by many authors are the following (see $[2,5]$ for example).

Condition 1. There is a constant $C_{R}$ such that the smoothing procedure satisfies:

$$
\frac{\|u\|^{2}}{\lambda} \leq C_{R}\left(R_{h} u, u\right) \quad \forall u \in \mathcal{M}_{h} .
$$

We note that (3) implies that

$$
\frac{\left\|A_{h} u\right\|^{2}}{\lambda} \leq C_{R} a\left(\left(I-K_{h}\right) u, u\right) \quad \forall u \in \mathcal{M}_{h} .
$$

For a multigrid algorithm, we require appropriate smoothers. We can use point and line Jacobi and Gauss-Seidel smoothing procedures to define $R_{h}$ (see [3] for example). If we set $R_{h}=\lambda^{-1}$, then with $C_{R}=1$, the equality holds in (3).

Condition 2 (regularity and approximation). For $\alpha \in(0,1]$

$$
a\left(\left(I-P_{h_{c}}\right) u, u\right) \leq C_{\alpha}^{2}\left(\frac{\left\|A_{h} u\right\|^{2}}{\lambda}\right)^{\alpha} a(u, u)^{1-\alpha} \quad \forall u \in \mathcal{M}_{h}
$$

where $C_{\alpha}$ is a constant independent of $h$ and $\lambda$ is the maximum eigenvalue of $A_{h}$.

Condition 3. The operator $H_{h_{c}}$ is symmetric in $a(.,$.$) inner products, i.e.$

$$
a\left(H_{h_{c}} v, w\right)=a\left(v, H_{h_{c}} w\right) \quad \forall v, w \in \mathcal{M}_{h_{c}}
$$

We also note that by induction, (6) implies that

$$
a\left(\widetilde{A}_{h_{c}}^{k} v, w\right)=a\left(v, \widetilde{A}_{h_{c}}^{k} w\right)
$$

for all $k \in \mathbf{N}$. 
The relation (6) holds for the Problem (2), since assume that $\operatorname{dim}\left(H_{h_{c}}\right)=$ $n$ and let $\phi_{1}, \cdots, \phi_{n}$ be an orthonormal basis for $\left(\mathcal{M}_{h_{c}},(., .)_{h_{c}}\right)$. For every $v \in \mathcal{M}_{h_{c}}$, we can write $v=\sum_{i=1}^{n} c_{i} \phi_{i}$. Then (see [8])

$$
H_{h_{c}} v=\sum_{i} \sum_{j} c_{j} b\left(\phi_{i}, \phi_{j}\right) \phi_{i}
$$

On the other hand,

$$
b\left(\phi_{i}, \phi_{j}\right)=\int_{\Omega} \int \phi_{i} . \phi_{j} d x=\left(\phi_{i}, \phi_{j}\right)_{h_{c}}=\delta_{i j}
$$

Hence, $H_{h_{c}}$ is an identity operator. Therefore,

$$
a\left(H_{h_{c}} v, w\right)=a(v, w)=a\left(v, H_{h_{c}} w\right) .
$$

\section{Main Results}

Our theory relates the convergence of multigrid algorithms to a "regularity and approximation " parameter $\alpha \in(0,1]$ and the number of relaxations $m$. In this section, we will present a convergence proof for Two-Grid Algorithm which has been stated in Section 2 .

The convergence rate for two-grid algorithm is measured by a convergence factor $\delta$ satisfying

$$
a\left(\left(I-B_{h} A_{h}\right) u, u\right) \leq \delta a(u, u), \quad \forall u \in \mathcal{M}_{h}
$$

for some $0<\delta<1$.

Nothing that $P_{h_{c}}^{0} A_{h}=A_{h_{c}} P_{h_{c}}$, similar to [2], it is straightforward to show that $B_{h}$ satisfies

$$
I-B_{h} A_{h}=K_{h}^{m}\left(I-B_{h_{c}} A_{h_{c}} P_{h_{c}}\right) K_{h}^{m} .
$$

We now turn to show inequality (8). First, we state the following lemmas.

Lemma 3.1 If $m_{1} \geq m_{2}$ where $m_{1}$ and $m_{2}$ are integers, then

$$
a\left(\left(I-K_{h}\right) K_{h}^{m_{1}} u, u\right) \leq a\left(\left(I-K_{h}\right) K_{h}^{m_{2}} u, u\right) .
$$

Proof: See [11].

Lemma 3.2 Let $d$ be a scaler. The series $\frac{1}{d} \sum_{i=0}^{n}\left(-\frac{1}{d} \widetilde{A}_{h_{c}}\right)^{i}$ converges if and only if $\rho\left(\widetilde{A}_{h_{c}}\right)<d$. Under this condition, the inverse of $d I+\widetilde{A}_{h_{c}}$ exists and the limit of the series is equal to $\left(d I+A_{h_{c}}\right)^{-1}$. 
Proof. It suffices to set $A:=\frac{-1}{d} \widetilde{A}_{h_{c}}$ in the proof of Theorem 1.5 in [10].

We recall that if an operator $T: M \rightarrow M$ is symmetric in arbitrary $s(.,$. inner product, then the following relation holds:

$$
\rho_{1} s(u, u) \leq s(T u, u) \leq \rho_{n} s(u, u), \quad, \forall u \in M .
$$

where $\rho_{1}$ and $\rho_{n}$ are minimum and maximum eigenvalues of $T$, respectively. Now, we are ready to state the following lemma.

Lemma 3.3 Let $\widetilde{A}_{h_{c}}=A_{h_{c}}+\sigma H_{h_{c}}$ which $\sigma$ is a nonnegative real number. Let also $\lambda_{1}, \mu_{1}$ and $\mu_{n}$ be the minimum eigenvalues of $A_{h_{c}}$, minimum and maximum eigenvalues of $H_{h_{c}}$, respectively. Then

$$
a\left(\widetilde{A}_{h_{c}}^{-1} H_{h_{c}} u, u\right) \leq \frac{\mu_{n}}{\lambda_{1}+\sigma \mu_{1}} a(u, u)
$$

holds for all $u \in \mathcal{M}_{h_{c}}$.

Proof. Let $d$ be a scaler and greater than maximum eigenvalues of $\widetilde{A}_{h_{c}}$. Then by Lemma 3.2,

$$
a\left(\widetilde{A}_{h_{c}}^{-1} H_{h_{c}} u, u\right)=\frac{1}{d} \sum_{i=0}^{\infty} a\left(\left(I-\frac{\widetilde{A}_{h_{c}}}{d}\right)^{i} H_{h_{c}} u, u\right)=\frac{1}{d} \sum_{i=0}^{\infty} a\left(\left(I-\frac{\widetilde{A}_{h_{c}}}{d}\right)^{i} u, H_{h_{c}} u\right)
$$

By (6), (7) and Holder's inequality,

$$
a\left(\widetilde{A}_{h_{c}}^{-1} H_{h_{c}} u, u\right) \leq \frac{1}{d} \sum_{i=0}^{\infty} a\left(\left(I-\frac{\widetilde{A}_{h_{c}}}{d}\right)^{2 i} u, u\right)^{1 / 2} a\left(H_{h_{c}}^{2} u, u\right)^{1 / 2}
$$

Then,

$$
a\left(\widetilde{A}_{h_{c}}^{-1} H_{h_{c}} u, u\right) \leq \frac{1}{d} \cdot \frac{d}{\lambda_{1}+\sigma \mu_{1}} a(u, u)^{1 / 2} \cdot \mu_{n} a(u, u)^{1 / 2}=\frac{\mu_{n}}{\lambda_{1}+\sigma \mu_{1}} a(u, u) .
$$

We now state and prove a theorem for estimating $\delta$ in (8) for Two-Grid Algorithm.

Theorem 3.4 Assume that Conditions (1), (2) and (3) hold. Define $B_{h}$ and $B_{h_{c}}$ by Two-Grid Algorithm. Then, for sufficiently small $\sigma$, the relation (8) holds with

$$
\sigma=\frac{\theta+1}{2}
$$

where $0<\theta=\frac{\mu_{n}}{\lambda_{1}+\sigma \mu_{1}}<1$ and $\lambda_{1}, \mu_{1}$ and $\mu_{n}$ are given as in Lemma 3.3. 
Proof. First, we note that

$$
I-B_{h_{c}} A_{h_{c}} P_{h_{c}}=\left(I-P_{h_{c}}\right)+\sigma \widetilde{A}_{h_{c}}^{-1} H_{h_{c}} P_{h_{c}} .
$$

On the other hand, since $K_{h}$ is symmetric in $a(.,$.$) , then by (9)$,

$$
a\left(\left(I-B_{h} A_{h}\right) u, u\right)=a\left(\left(I-B_{h_{c}} A_{h_{c}} P_{h_{c}}\right) K_{h}^{m} u, K_{h}^{m} u\right) .
$$

Now, by (11) and (12),

$$
a\left(\left(I-B_{h} A_{h}\right) u, u\right)=a\left(\left(I-P_{h_{c}}\right) K_{h}^{m} u, K_{h}^{m} u\right)+\sigma a\left(\widetilde{A}_{h_{c}}^{-1} H_{h_{c}} P_{h_{c}} K_{h}^{m} u, K_{h}^{m} u\right) .
$$

By definition of $P_{h_{c}}$,

$$
a\left(\left(I-B_{h} A_{h}\right) u, u\right) \leq a\left(\left(I-P_{h_{c}}\right) K_{h}^{m} u, K_{h}^{m} u\right)+\sigma a\left(\widetilde{A}_{h_{c}}^{-1} H_{h_{c}} P_{h_{c}} K_{h}^{m} u, P_{h_{c}} K_{h}^{m} u\right) .
$$

Now, by Lemma 3.3

$$
a\left(\left(I-B_{h} A_{h}\right) u, u\right) \leq a\left(\left(I-P_{h_{c}}\right) K_{h}^{m} u, K_{h}^{m} u\right)+\frac{\sigma \mu_{n}}{\lambda_{1}+\sigma_{1} \mu_{1}} a\left(P_{h_{c}} K_{h}^{m} u, K_{h}^{m} u\right) .
$$

Then,

$$
\begin{array}{r}
a\left(\left(I-B_{h} A_{h}\right) u, u\right) \leq a\left(\left(I-P_{h_{c}}\right) K_{h}^{m} u, K_{h}^{m} u\right)+\theta a\left(P_{h_{c}} K_{h}^{m} u, K_{h}^{m} u\right)= \\
(1-\theta) a\left(\left(I-P_{h_{c}}\right) K_{h}^{m} u, K_{h}^{m} u\right)+\theta a\left(K_{h}^{m} u, K_{h}^{m} u\right) .
\end{array}
$$

On the other hand, by Lemma (6.1) in [7] and (5)

$a\left(\left(I-P_{h_{c}}\right) K_{h}^{m} u, K_{h}^{m} u\right) \leq C_{\alpha}^{2}\left\{\alpha \gamma^{-1 / \alpha}\left(\frac{\left\|A_{h} K_{h}^{m} u\right\|^{2}}{\lambda}\right)+(1-\alpha) \gamma^{1 /(1-\alpha)} a\left(K_{h}^{m} u, K_{h}^{m} u\right)\right\}$

holds for any positive $\gamma$.

We choose $\sigma<\frac{\lambda_{1}}{\mu_{n}-\mu_{1}}$ and so $\theta<1$. The relations (4), (13) and Lemma 3.1 imply that

$$
\begin{aligned}
a\left(\left(I-B_{h} A_{h}\right) u, u\right) \leq & {\left[(1-\theta)(1-\alpha) C_{\alpha}^{2} \gamma^{\frac{1}{1-\alpha}}+\theta\right] a\left(K_{h}^{2 m} u, u\right) } \\
& +(1-\theta) C_{\alpha}^{2} \frac{\alpha}{2 m} C_{R} \gamma^{\frac{-1}{\alpha}} a\left(\left(I-K_{h}^{2 m}\right) u, u\right) .
\end{aligned}
$$

To prove that (8) holds, we can choose $\gamma$ so that

$$
(1-\theta)(1-\alpha) C_{\alpha}^{2} \gamma^{\frac{1}{1-\alpha}}+\theta \leq \delta
$$


and

$$
(1-\theta) C_{\alpha}^{2} \frac{\alpha}{2 m} C_{R} \gamma^{\frac{-1}{\alpha}} \leq \delta .
$$

Then, $\gamma$ must satisfies the following inequalities:

$$
\left(\frac{(1-\theta) C_{\alpha}^{2} \frac{\alpha}{2 m} C_{R}}{\delta}\right)^{\alpha} \leq \gamma \leq\left(\frac{\delta-\theta}{(1-\theta)(1-\alpha) C_{\alpha}^{2}}\right)^{1-\alpha}
$$

We choose $\delta>\theta$. If we set

$$
e=C_{\alpha}^{2}\left(\frac{\alpha}{2 m} C_{R}\right)^{\alpha}(1-\alpha)^{1-\alpha},
$$

then $\delta$ satisfies

$$
\delta^{\alpha}(\delta-\theta)^{1-\alpha} \geq e(1-\theta) .
$$

Choosing $\delta=\frac{\theta+1}{2}$, we have

$$
\left(\frac{1+\theta}{1-\theta}\right)^{\alpha} \geq 2 e
$$

The relation (17) clearly holds for sufficiently large $m$. Now, if we choose $\gamma$ to be the average of

$$
\left(\frac{(1-\theta) C_{\alpha}^{2} \frac{\alpha}{2 m} C_{R}}{\delta}\right)^{\alpha}
$$

and

$$
\left(\frac{\delta-\theta}{(1-\theta)(1-\alpha) C_{\alpha}^{2}}\right)^{1-\alpha} .
$$

then, the proof of Theorem 3.4 is completed.

Remark 1. It can be shown that on the periodic domain, $C_{\alpha}^{2}\left(h_{k}\right) \leq$ $4+2 \sqrt{2}=6.828 \ldots$ for $\alpha=1$ (see [2]). Then for $m \geq 7$ the multigrid algorithm would be convergent.

\section{References}

[1] J. Bramble, Multigrid Methods, Pitman, London, 1993.

[2] J. Bramble and J. Pasciak, New convergence estimates for multigrid algorithms, Math. Comp., 49(1987), 311-329.

[3] J. Bramble and J. Pasciak, The analysis of smoothers for multigrid algorithms, Math. Comp., 58(1992), 467-488.

[4] J. Bramble and J. Pasciak, New estimates for multilevel algorithms including the V-cycle, Math. Comp., 60(1993), 447-471. 
[5] J. Bramble, J. Pasciak and J. Xu, The analysis of multigrid algorithms with non-nested spaces or non-inherited quadratic forms, Math. Comp., 56(1991), 1-34.

[6] W. L. Briggs, V. E. Henson and S. F. McCormick, A multigrid tutorials, Second edition, Siam, 2000.

[7] G. B. Folland, Real analysis, modern techniques and their applications, Second edition, John Wiley and \& Sons, INC, 1999.

[8] K. Hoffman and R. Kunze, Linear algebra, Second edition, Prentice-Hall, 1971.

[9] S. Kim, Artificial damping in multigrid methods, App. Math. Letters, 14(2001), 359-364.

[10] Y. Saad, Iterative methods for sparse linear system, Second edition, Society for industrial and applied, 2003.

[11] A. Tavakoli and A. Kerayechian, Improvement of the rate of convergence estimates for multigrid algorithm, App. Math. and Comp., 187(2007), 922-928.

Received: August 12, 2007 